been referred and appointments booked pre-COVID but urgent (2 -week) referrals from GPs were also seen.

Patients/parents/carers were called at least 3 times on 3 different occasions over at least 2 days. If they did not answer they were listed as 'was not brought'. Calls were made between 9 am to $5 \mathrm{pm}$ Monday to Friday.

Results 262 new patients had new General Paediatric Clinic appointments and 235 (90\%) were successfully contacted. 27 (10\%) of patients 'were not brought'. These 27 patients were excluded from on-going analysis.

Following one telephone consultation, 105 (45\%) contacted patients could be discharged from the General Paediatric clinic with parental agreement. Of these 27 (11\%) patients had a complaint which had resolved by the time of the appointment and $19(8 \%)$ patients had already been seen by a private consultant or other NHS consultant. Thus 46 (20\%) of the contacted patients did not need their General Paediatric appointment.

Only 7 (3\%) patients needed urgent face-to face-review of which 4 were urgent 2-week wait referrals by the GP. 155 (66\%) patients needed no investigations.

Conclusions The NHS is going through unchartered, challenging time. However, this provides opportunities to adapt and evolve services, improving efficiency of care for both the families we look after and health service staff. The data above highlights that almost half of the new General Paediatric outpatients could be successfully discharged from clinic after one telephone consultation. During a time of significant anxiety and uncertainty families avoided hospital contact. A similar system when we return to normality could lead to fewer absences from school and less time off work for parents. Out experience suggests that initial telephone consultations with new General Paediatric patients may be an efficient way to make first patient contact.

\section{CLINICAL CORRELATES OF CORD ARTERIAL LACTATE IN TERM BABIES}

Pei Sen Tee. UK

\subsection{6/bmjpo-2021-RCPCH.29}

Background Umbilical cord blood gas analysis is recommended to be taken when there has been concern about the baby in labour or immediately following birth and cord arterial lactate (CAL) has become a standard measurement on blood gas reports. However, evidence regarding thresholds for concern or appropriate management remains limited. Within our unit, guidance on further management of infants following detection of potentially 'raised' CAL was ratified based on available literature.

Objectives This study reports on the clinical correlates of a raised cord arterial lactate following introduction of a guidance to manage newborns with raised CAL.

Methods A single-centre retrospective review of all term neonates (>35 weeks gestational age) with a recorded CAL over a 6-month period (July 2018 to December 2018). Babies were identified from maternity monthly statistics and data extracted from electronic medical records. Clinical course and adverse events were evaluated between risk groups: Low-risk CAL $\leq 5$ $\mathrm{mmol} / \mathrm{L}$, moderate-risk CAL 5-10 $\mathrm{mmol} / \mathrm{L}$ and high-risk CAL $\geq 10 \mathrm{mmol} / \mathrm{L}$. Adverse events include therapeutic hypothermia,

\begin{tabular}{|c|c|c|}
\hline $\begin{array}{l}\text { CAL } \\
\text { (mmol/L) }\end{array}$ & Immediate Action & $\begin{array}{l}\text { Further management/ } \\
\text { investigations }\end{array}$ \\
\hline$<5$ & $\begin{array}{l}\text { Baby to stay with mother. } \\
\text { 'Routine' postnatal care }\end{array}$ & No follow-up \\
\hline $5-10$ & $\begin{array}{l}\text { Baby to stay with mother, keep } \\
\text { warm, encourage early breastfeeding. }\end{array}$ & $\begin{array}{l}\text { - 4-hourly observations on postnatal } \\
\text { ward for } 24 \text { hours. } \\
\text { - Consider repeat blood gas in 4-6 } \\
\text { hours from well perfused heel }\end{array}$ \\
\hline$>10$ & $\begin{array}{l}\text { Consider admission to unit, cerebral } \\
\text { function monitoring and careful } \\
\text { neurological assessment }\end{array}$ & $\begin{array}{l}\text { - Close observation including regular } \\
\text { blood gases and blood glucose } \\
\text { monitoring. } \\
\text { - Discussion with senior clinician } \\
\text { regarding plans (feeding). }\end{array}$ \\
\hline
\end{tabular}

mechanical ventilation, neonatal death and usage of non-invasive ventilation.

Results Among 2591 infants born $\geq 35$ weeks gestation, 658 (25\%) had a recorded CAL $(44 \%$ had a CAL $<5 \mathrm{mmol} / \mathrm{L}$, $49 \%$ had CAL $5-10 \mathrm{mmol} / \mathrm{L}$ and $7 \%$ had CAL $\geq 10 \mathrm{mmol} / \mathrm{L}$ ). Gender gestation and birth weight were in equal proportions in all 3 groups. The high-risk group with $\mathrm{CAL} \geq 10 \mathrm{mmol} / \mathrm{L}$ required more resuscitation at birth, NICU admission and had more adverse events compared to groups with $\mathrm{CAL} \leq 10$ $\mathrm{mmol} / \mathrm{L}$. There was no difference in these events in infants where $\mathrm{CAL}<5 \mathrm{mmol} / \mathrm{L}$ compared to those with a CAL 5-10 $\mathrm{mmol} / \mathrm{L}$.

A repeat blood lactate level was taken in $48 \%$ and $98 \%$ of CAL $5-10 \mathrm{mmol} / \mathrm{L}$ and $\mathrm{CAL} \geq 10 \mathrm{mmol} / \mathrm{L}$ groups respectively. The time range for first repeat varied widely and babies who had increased lactate level after repeat did not receive more treatment than those where the lactate fell. Routine repeat of blood lactate levels in clinically well babies with CAL 5-10 $\mathrm{mmol} / \mathrm{L}$ did not appear to change management.

Conclusions A large number of babies are currently labelled as moderate to high risk based on a single raised CAL which may lead to unnecessary medical interventions. Better evidence is needed to determine the significance of a raised CAL, define clinically important thresholds for concern and optimal interventional strategies.

\section{MEDICATION ERRORS IN PAEDIATRICS DEPARTMENT AND THEIR IMPACT ON PATIENT SAFETY}

Pei Sen Tee. UK

\subsection{6/bmjpo-2021-RCPCH.30}

Background Medication errors (MEs) represent a significant burden to healthcare system and the frequency of potentially harmful MEs was found to be three times higher in paediatric population when compared to adults ${ }^{1}$.

Objectives This review aims to analyse MEs to improve medication safety and compare the frequency of MEs compared to the previous audit cycle.

Methods This is a retrospective analysis of medication errors in paediatric ward and neonatal unit utilising critical incident reporting forms (Datix) that were submitted from October 2018 to September 2019. These reports were obtained 
through the clinical audit department and patients' case notes were examined to review attributes of error.

Results There were 38 incidents over the period of review (3.2 errors per month), demonstrating an improvement from the last audit cycle (4.5 errors per month). A large percentage $(68 \%)$ of errors originated from paediatric ward and most of the errors were attributable to prescription errors. Doctors of all grades were identified to commit errors in prescription. Administration issues were the second most common cause for errors. Neither of these errors resulted in mortality and morbidity although some patients required additional monitoring as a consequence.

Conclusions It is important to note that while errors were reducing in frequency, these errors could still potentially cause harm if they were not being addressed. Our trust is currently in the process of rolling out electronic prescribing and administration of medications to reduce medication errors and improve patient care. Another audit will be planned after the implementation of electronic system to review its usage to improve medication safety.

\section{MAXIMISING THE EDUCATIONAL VALUE OF CLERKING FOR JUNIOR TRAINEES ON A PAEDIATRIC CARDIOLOGY WARD}

Emily Operto, Esme Dunne. UK

\subsection{6/bmjpo-2021-RCPCH.31}

Background The cardiorespiratory physiology of congenital heart defects can be complex and junior trainees infrequently receive training in echocardiogram interpretation. A rotation in paediatric cardiology provides a wealth of learning opportunities however these may not be realised because of service pressures.

Objectives This quality improvement project aimed to maximise the educational opportunities of clerking ward admissions for junior trainees on a paediatric cardiology ward. The two structured interventions focussed on tandem interpretation of patients' cardiorespiratory physiology and echocardiogram findings, between junior and senior trainees, with the aim of encouraging learning and feedback.

Methods An anonymous questionnaire of six junior trainees collected baseline quantitative data to assess educational aspects of clerking admissions on a cardiology ward in a paediatric cardiology centre in London. Two educational interventions were implemented for 4 months and the questionnaire was subsequently repeated. The first educational intervention was a poster reminding junior trainees to draw a box heart and to review the echocardiogram for the newly admitted patients they clerked. The second educational intervention was a junior-senior discussion on the cardiorespiratory pathophysiology and key findings on echocardiogram for the newly admitted patients they clerked. The results were analysed using percentages.

Results Following the implementation of the two interventions, the proportion of junior trainees who drew a box heart and reviewed the echocardiogram of patients they clerked increased. Junior trainees reported a higher level of confidence in their understanding of the cardiorespiratory physiology of patients they clerked, and in identifying their key findings on echocardiogram. The reported combined time taken to draw a box heart and review the echocardiogram did not exceed fifteen minutes.

Conclusions Drawing a box heart and reviewing the echocardiogram with senior support are two quick methods to increase the educational aspects of clerking ward admissions for junior trainees on a paediatric cardiology ward.

\section{THE KEY BARRIERS TO THE UPTAKE OF PMTCT SERVICES IN UGANDA}

Emily Operto. UK

\subsection{6/bmipo-2021-RCPCH.32}

Background Mother-to-child transmission of the human immunodeficiency virus (HIV) is the second leading cause of new HIV infections among children in Uganda. However, approximately $40-50 \%$ of eligible women in Uganda do not use 3 of the key services provided by a programme of interventions to prevent this route of HIV transmission (PMTCT). A report on the global success of the prevention of mother-to-child transmission programme found that barriers exist at individual community levels to accessing these services.

Objectives The aim of the review is to explore the key barriers to the uptake of services in component 3 of the prevention of mother-to-child transmission programme by women in Uganda.

Methods A systematic, focussed literature search was carried out using relevant databases. Inclusion criteria were used to select relevant studies.

Results The review identified a number of key barriers that affected uptake of services provided by component 3. Gender norms limit the ability of HIV-positive women to make decisions about accessing and accepting HIV testing and anti-retroviral prophylaxis. HIV-related stigma poses a significant deterrent from accessing both of these services, and adhering to anti-retroviral regimens. Low quality of HIV and infant feeding counselling may affect acceptance and adherence to HIV testing and anti-retroviral prophylaxis. A limited amount of literature was available discussing factors that influenced infant feeding practices among HIV-positive mothers however studies which had been carried out suggested that cultural norms are the strongest barrier to the acceptance of exclusive breastfeeding or exclusive replacement feeding for the first 6 months of the infant's life.

Conclusions Women eligible for the prevention of mother-tochild transmission programme are in need of further support to improve their access and adherence to services. A number of strategies have been suggested to address these barriers.

\section{CLINICAL AUDIT ON THE MANAGEMENT OF KAWASAKI DISEASE}

Emily Operto, Filip Kucera. UK

\subsection{6/bmjpo-2021-RCPCH.33}

Background Kawasaki disease is a systemic vasculitis of unknown etiology that can result in coronary artery aneurysms in up to $25 \%$ of patients. ${ }^{2}$ This an important cause of morbidity and mortality in this condition. ${ }^{1}$ In the longer term, patients with persistent coronary artery aneurysms can develop 